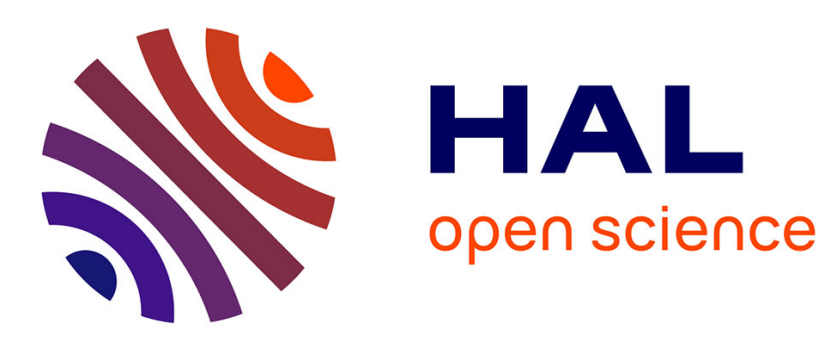

\title{
Object-Oriented Database Benchmarks
}

Jérôme Darmont, Michel Schneider

\section{To cite this version:}

Jérôme Darmont, Michel Schneider. Object-Oriented Database Benchmarks. Advanced Topics in Database Research, 1, Idea Group Publishing, pp.34-57, 2002, 10.4018/978-1-930708-41-9.ch003 . hal-01435501

\section{HAL Id: hal-01435501 https://hal.science/hal-01435501}

Submitted on 14 Jan 2017

HAL is a multi-disciplinary open access archive for the deposit and dissemination of scientific research documents, whether they are published or not. The documents may come from teaching and research institutions in France or abroad, or from public or private research centers.
L'archive ouverte pluridisciplinaire HAL, est destinée au dépôt et à la diffusion de documents scientifiques de niveau recherche, publiés ou non, émanant des établissements d'enseignement et de recherche français ou étrangers, des laboratoires publics ou privés. 


\title{
Object-Oriented Database Benchmarks
}

\author{
Jérôme Darmont ${ }^{1}$, Michel Schneider ${ }^{2}$ \\ ${ }^{1}$ Laboratoire ERIC \\ Université Lumière - Lyon 2 \\ 5 avenue Pierre Mendès-France \\ 69676 Bron Cedex \\ FRANCE \\ ${ }^{2}$ Laboratoire LIMOS \\ Université Blaise Pascal - Clermont-Ferrand II \\ Complexe Scientifique des Cézeaux \\ 63177 Aubière Cedex \\ FRANCE
}

Contact e-mail: jerome.darmont@univ-lyon2.fr

Phone: (33) 478774492

Fax: (33) 478772375

Abstract: We present in this chapter an overview of the benchmarks aimed at evaluating the performances of Object-Oriented Databases (OODBs). We particularly focus on OCB (the Object Clustering Benchmark), which is both generic and originally designed to specifically evaluate the performances of clustering policies within OODBs. OCB is generic because its sample database may be customized to fit any of the databases introduced by the main previous benchmarks, e.g., OO1 (Object Operation 1) or OO7. The first version of OCB was purposely clustering-oriented due to a clustering-oriented workload, but OCB has been thoroughly extended to be able to suit other purposes. Eventually, OCB's code is compact and easily portable. $O C B$ has been validated through two implementations: one within the $\mathrm{O}_{2}$ $O O D B$ and another one within the Texas persistent object store. The performances of two different clustering policies implemented in Texas, DSTC (Dynamic, Statistical, Tunable Clustering) and DRO (Detection \& Reclustering of Objects), have also been compared with OCB. Keywords: Object-Oriented Databases, Benchmarking, Performance Evaluation, Clustering.

\section{INTRODUCTION}

The need to evaluate the performances of Object-Oriented Database Management Systems (OODBMSs) is important both to designers and users. Performance evaluation is useful to designers to determine elements of architecture, choose between caching strategies, and select 
Object Identifier (OID) type, among others. It helps them validate or refute hypotheses regarding the actual behavior of an OODBMS. Thus, performance evaluation is an essential component in the development process of efficient and well-designed object stores. Users may also employ performance evaluation, either to compare the efficiency of different technologies before selecting an OODBMS or to tune a system.

The main work presented in this chapter was initially motivated by the evaluation of object clustering techniques. The benefits induced by such techniques on global performances are widely acknowledged and numerous clustering strategies have been proposed. As far as we know, there is no generic approach allowing for their comparison. This problem is interesting for both designers (to set up the corresponding functionalities in the system kernel) and users (for performance tuning).

There are different approaches used to evaluate the performances of a given system: experimentation, simulation, and mathematical analysis. We focus only on the first two approaches. Mathematical analysis is not considered because it invariably uses strong simplification hypotheses (Benzaken, 1990; Gardarin et al., 1995) and its results may well differ from reality. Experimentation on the real system is the most natural approach and a priori the simplest to complete. However, the studied system must have been acquired, installed, and have a real database implanted in it. This database must also be significant of future exploitation of the system. Total investment and exploitation costs may be quite high, which can be drawbacks when selecting a product.

Simulation is casually used in substitution or as a complement to experimentation. It does not necessitate installing nor acquiring the real system. It can even be performed on a system still in development (a priori evaluation). The execution of a simulation program is generally much faster than experimentation. Investment and exploitation costs are very low. However, this approach necessitates the design of a functioning model for the studied system. The reliability of results directly depends on the quality and the validity of this model. Thus, the main difficulty is to elaborate and validate the model. A modelling methodology can help and secure these tasks.

Experimentation and simulation both necessitate a workload model (database and operations to run on this database) and a set of performance metrics. These elements are traditionally provided by benchmarks. Though interest for benchmarks is well recognized for experimentation, simulation approaches usually use workloads that are dedicated to a given study, rather than workloads suited to performance comparisons. We believe that benchmarking techniques 
can also be useful in simulation. Benchmarking can help validate a simulation model as compared to experimental results or support a mixed approach in which some performance criteria necessitating precision are measured by experimentation and other criteria that does not necessitate precision are evaluated by simulation.

There is no standard benchmark for OODBs, even if the more popular benchmarks, OO1, HyperModel, and $\mathrm{OO} 7$ are de facto standards. These benchmarks are aimed at engineering applications (Computer Aided Design, Manufacturing, or Software Engineering). These general-purpose benchmarks feature quite simple databases and are not well suited to the study of clustering, which is very data-dependent. Many benchmarks have been developed to study particular domains. A fair number of them are more or less based on OO1, HyperModel, or OO7.

In order to evaluate the performances of clustering algorithms within OODBs, a new benchmark was designed: OCB (Darmont et al., 1998). It originally had a generic object base and was clustering-oriented through its workload. It actually appeared afterwards that OCB could become more general, provided the focused workload was extended, as described in this chapter.

Full specifications for a new version of OCB are provided in this chapter. More precisely, we address the following points: the generalization of the OCB workload so that the benchmark becomes fully generic, a comparison of OCB to the main existing benchmarks, and a full set of experiments performed to definitely validate OCB. These performance tests were performed on the $\mathrm{O}_{2}$ OODB (Deux, 1991), the Texas persistent object store (Singhal et al., 1992), and the DSTC (Bullat \& Schneider, 1996) and the DRO (Darmont et al., 2000) clustering techniques, that are both implemented in Texas. The results obtained are discussed in this chapter.

We are aware of the legal difficulties pointed out by Carey et al. (1993) and Carey et al. (1994). Indeed, OODBMS vendors are sometimes reluctant to see benchmark results published. The objective of our effort is rather to help software designers or users evaluate the adequacy of their product in a particular environment. OCB should also prove useful at least for researchers, to benchmark OODB prototypes and/or evaluate implementation techniques. The remainder of this chapter is organized as follows. The de facto standards in objectoriented benchmarking are presented (OO1, HyperModel, and OO7; as well as the Justitia benchmark, which is interesting due to its multi-user approach). Next, the OCB benchmark is 
described and compared to the other benchmarks. Experiments performed to validate OCB are also presented. The chapter is concluded with future trends.

\section{STATE OF THE ART}

The OO1 Benchmark

OO1, also referred to as the "Cattell Benchmark" (Cattell, 1991), was developed early in the 1990's when there was no appropriate benchmark for engineering applications. OO1 is a simple benchmark that is very easy to implement. It was used to test a broad range of systems including object-oriented DBMS, relational DBMS, and other systems such as Sun's INDEX (B-tree based) system. The visibility and simplicity of OO1 provide a standard for OODB benchmarking. A major drawback of this tool is that its data model is too elementary to measure the elaborate traversals that are common in many types of object-oriented applications, including engineering applications. Furthermore, OO1 only supports simple navigational and update tasks and has a limited notion of complex objects (only one composite hierarchy).

\section{OO1 Database}

OO1's database (Figure 1) is built upon two classes: Part and Connection. The parts are composite elements that are connected (through Connection objects) to three other parts. Each connection references two parts: the source (From) and the destination part (To).

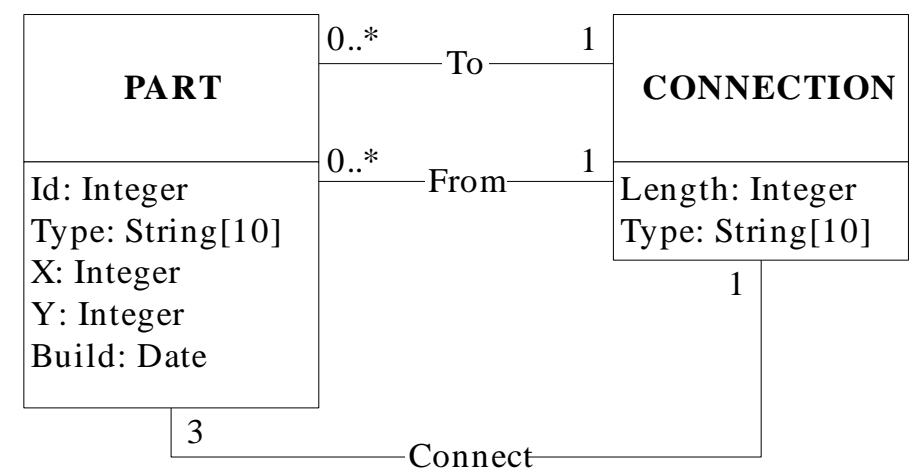

Figure 1: OOl database schema

The database is generated as follows: 
1. create all the Part objects and store them into a dictionary;

2. for each part, randomly choose three other parts and create the associated connections.

Locality of reference (objects are often linked to relatively close objects) is simulated by a reference zone. I.e., Part \#i is randomly linked to parts whose $I d$ are in the interval [IdRefZone, Id+RefZone]. The probability that the links are determined this way is 0.9. Otherwise, the linked parts are chosen totally at random.

\section{OO1 Operations}

OO1 performs three types of operations. Each of them is run 10 times. Response time is measured for each run.

- Lookup: access to 1000 randomly selected parts.

- Traversal: randomly select a root part, then explore the corresponding part tree (in depth first) through the Connect and To references, up to seven hops (total of 3280 parts, with possible duplicates). Also perform a reverse traversal by swapping the To and From directions.

- Insert: add 100 parts, and the corresponding connections, to the database. Commit the changes.

The HyperModel Benchmark

The HyperModel Benchmark (Anderson et al., 1990), also referred to as the Tektronix Benchmark, is recognized for the richness of the tests it proposes. HyperModel possesses both a richer schema and a wider extent of operations than OO1. This renders it potentially more effective than $\mathrm{OO} 1$ in measuring the performances of engineering databases. However, this added complexity also makes HyperModel harder to implement, especially since its specifications are not as complete as OO1's. The presence of complex objects in the HyperModel Benchmark is limited to a composition hierarchy and two inheritance links. The scalability of HyperModel is also not clearly expressed in the literature, whereas other benchmarks explicitly support different and well identified database sizes.

HyperModel Database 
The HyperModel Benchmark is based on an extended hypertext model. Hypertext is a generic graph structure consisting of nodes and links (Figure 2). The main characteristic of this database is the various relationships existing between classes: inheritance (the attributes of a Node object may be inherited from another Node object), aggregation (an instance of the Node class may be composed of one or several other instances), and eventually association (two Node objects may be bound by an oriented link).

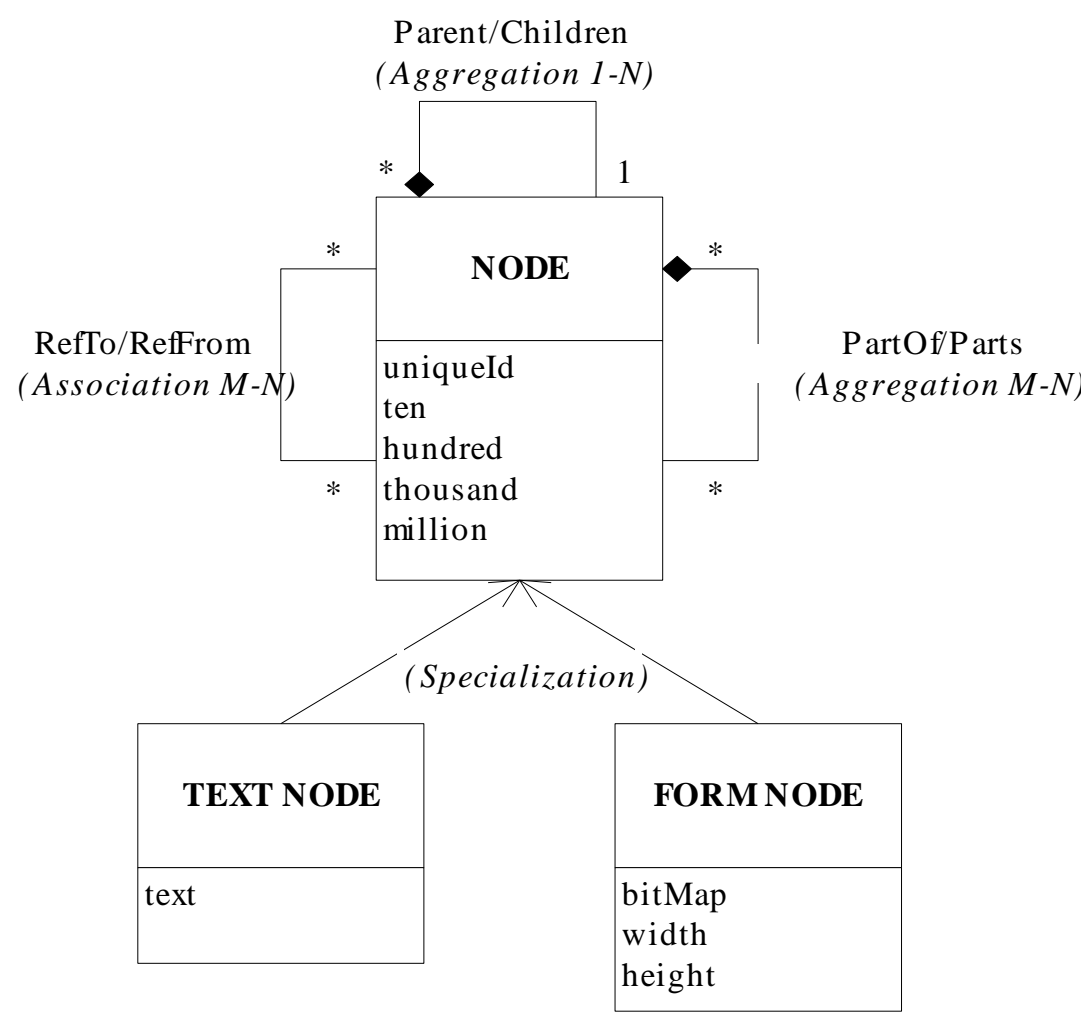

Figure 2: HyperModel database schema

\section{HyperModel Operations}

The benchmark consists of 20 operations. To measure the time to perform each operation, the following sequence is followed.

1. Setup: prepare 50 inputs to the operations (the setup is not timed);

2. Cold run: run the operation 50 times, on the 50 inputs precomputed in the setup phase; then, if the operation is an update, commit the changes once for all 50 operations;

3. Warm run: repeat the operation 50 times with the same input to test the effect of caching; again, perform a commit if the operation was an update. 
The 20 possible operations belong to seven different kinds:

- Name Lookup: retrieve one randomly selected node;

- Range Lookup: retrieve the nodes satisfying a range predicate based on an attribute value;

- Group Lookup: follow the relationships one level from a randomly selected starting node;

- Reference Lookup: reverse Group Lookup;

- Sequential Scan: visit all the nodes;

- Closure Traversal: Group Lookup up to a predefined depth;

- Editing: update one node.

The OO7 Benchmark

OO7 (Carey et al., 1993) is a more recent benchmark than OO1 and HyperModel. It reuses their structures to propose a more complete benchmark and to simulate various transactions running on a diversified database. It has also been designed to be more generic than its predecessors and to correct their weaknesses in terms of object complexity and associative accesses. This is achieved with a rich schema and a comprehensive set of operations.

However, if $\mathrm{OO} 7$ is a good benchmark for engineering applications, it is not the case for other types of applications such as financial, telecommunication, and multimedia applications (Tiwary et al., 1995). Since its schema is static, it cannot be adapted to other purposes. Eventually, the database structure and operations of $\mathrm{OO} 7$ are nontrivial. Hence, the benchmark is quite difficult to understand, adapt, or even implement. Yet, to be fair, OO7 implementations are available by anonymous FTP $^{1}$.

\section{OO7 Database}

OO7's database is based on a conceptual model that is very close to the HyperModel Benchmark's, though it contains a higher number of classes (Figure 3). Four kinds of links are also supported (IS-A, 1-1, 1-N, M-N).

\footnotetext{
${ }^{1} \mathrm{ftp}: / / \mathrm{ftp} . c s . w i s c . e d u / O O 7$
} 


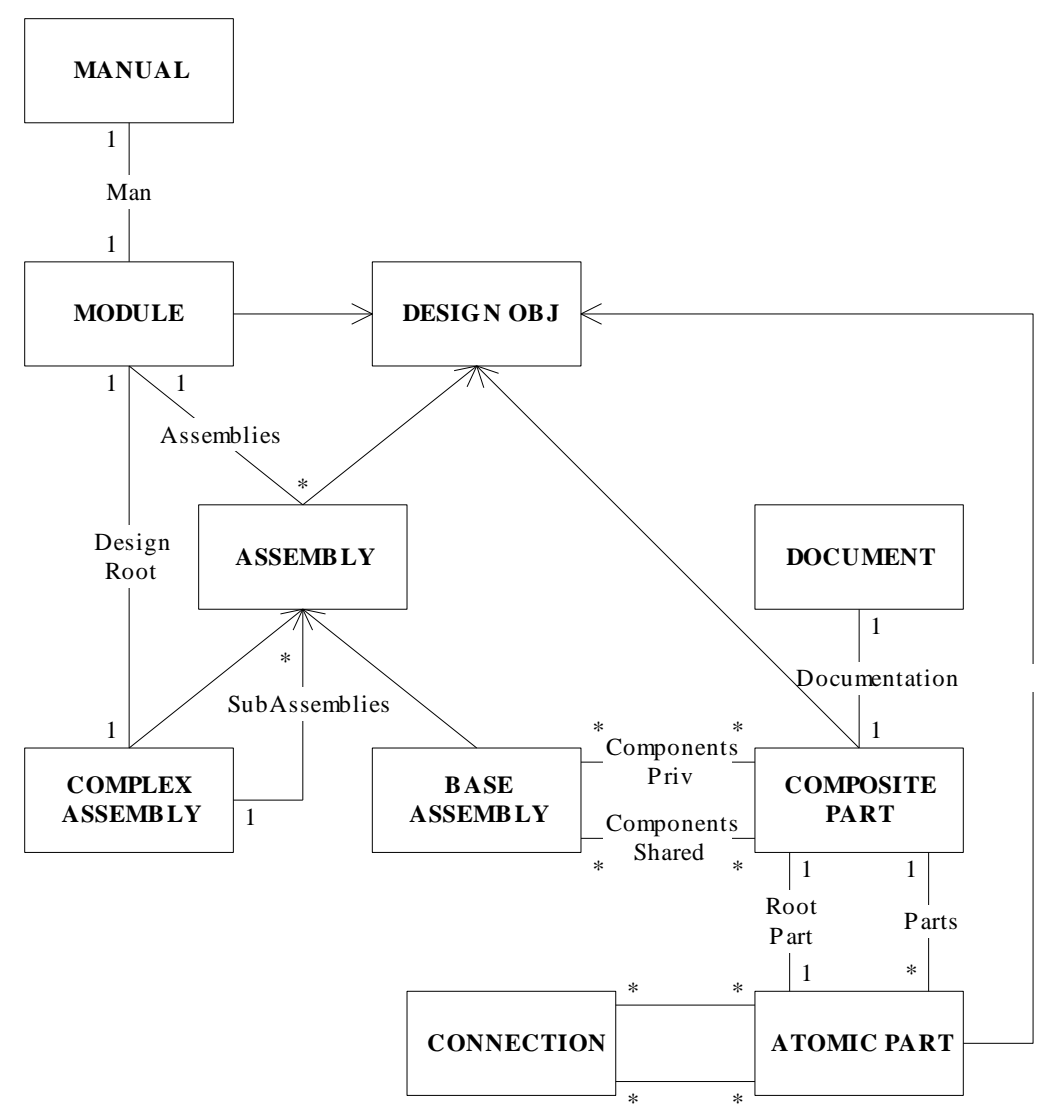

Figure 3: OO7 database schema

\section{OO7 Operations}

The range of transactions offered by $\mathrm{OO} 7$ is also close to HyperModel's. Three main groups may be identified:

- Traversals browse the object graph using certain criteria. These traversals are very close to OO1's. There are ten different operations that apply depending on the database characteristics (basically, its size);

- Queries retrieve objects chosen in function of various criteria. There are eight kinds of queries;

- Structural Modification Operations deal with object insertion and deletion (two operations).

The Justitia Benchmark 
Justitia (Schreiber, 1994) has been designed to address the shortcomings of existing benchmarks regarding multi-user functionality, which is important in evaluating client-server environments. Justitia is also aimed at testing OODB capacity in reorganizing its database.

Because Justitia's published specifications lack precision, the author's work cannot be easily reused. Furthermore, taking multiple users into account renders the benchmark quite complex. Justitia is fairly tunable and supposed to be generic, but it still uses structures that are typical of engineering applications. Its database schema is more limited than those of HyperModel or O07. Though the object types are diverse, inter-class relationships are very few. The inheritance graph is substantial, but other types of references are limited to composition.

\section{Justitia Database}

Justitia's database structure is a tree of bi-directional 1-N relationships interleaved with an inheritance graph (Figure 4). The basic classes that compose it are inspired by engineering application data structures:

- Dynamic-sized objects (DO) have a random size, which immunizes the benchmark against OODBs performing optimizations regarding a given object size;

- Static-sized objects (SO) represent typical objects; their size is available to the system, that can use it for optimization purposes;

- Container objects $(\mathrm{CO})$ are nodes pointing each to a ring of references toward primitive objects (PO) that represent complex, internal objects.

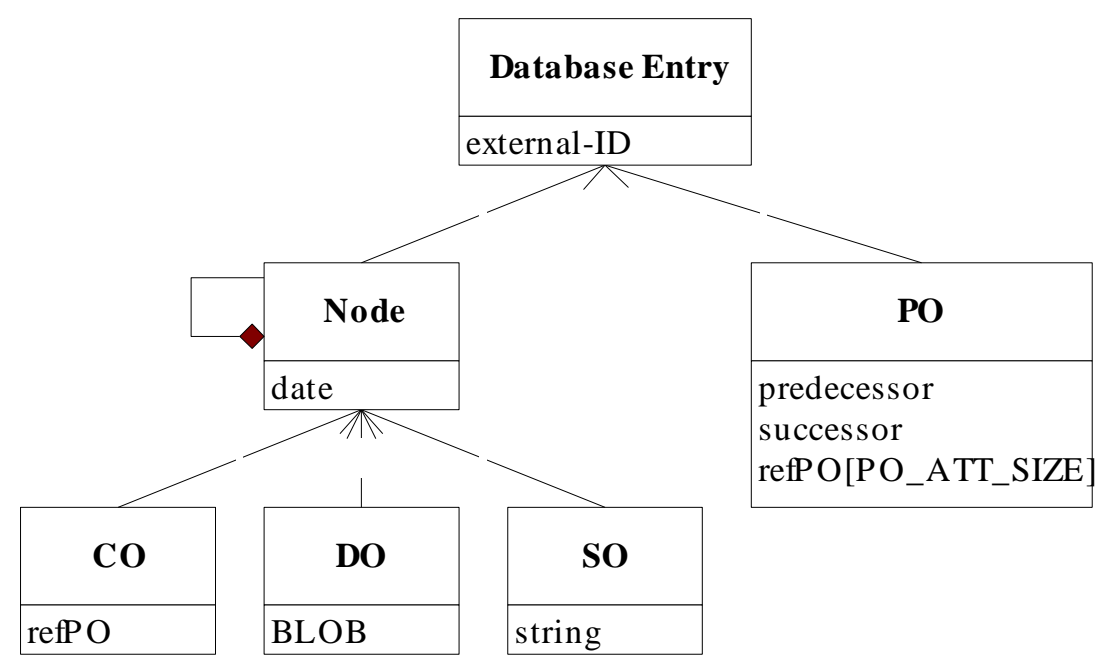

Figure 4: Justitia database schema 


\section{Justitia Operations}

All the benchmark operations are based on four basic transactions that can be combined to form composite operations. The basic operations use elementary access methods and model simple accesses.

- Simple read: Access a set of objects and read their date attribute.

- Simple update: Access a set of objects and update their date attribute.

- Extended read: Access a set of objects, read their date attribute, and execute an operation depending on their class.

- Extended update: Access a set of objects, update their date attribute, and execute an operation depending on their class.

The composite operations are defined by the user through a series of parameters. Their aim is to test the efficiency of the multi-user mode.

\section{THE OBJECT CLUSTERING BENCHMARK}

Originally, the purpose of OCB was to test the performances of clustering algorithms within object-oriented systems. OCB is structured around a rich object base including many different classes and numerous types of references allowing the design of multiple interleaved hierarchies. This database is wholly generic. The OCB workload, once clustering-oriented, has been extended with relevant, significant, and reproducible transactions. Thus, the workload became fully generic.

The flexibility of OCB is achieved through an extensive set of parameters. Many different kinds of object bases can be modeled with OCB as well as many different kinds of applications running on these databases. This is an important feature since there exists no canonical OODB application. OCB can indeed be easily parameterized to model a generic application or dedicated to a given type of object base and/or application. OCB is also readily scalable in terms of size and complexity resulting in a wide range of object bases. Usage time can be set up as well to be rather short or more extensive. Moreover, OCB's parameters are easy to set up. 
OCB's code is very compact and easily implemented on any platform. OCB is currently implemented in $\mathrm{C}++$ to benchmark $\mathrm{O}_{2}$ and Texas. Both versions are freely available ${ }^{2}$. The $\mathrm{C}++$ code is less than 1,500 lines long. OCB has also been ported into QNAP2 and C++ simulation models. QNAP2 is a simulation software that supports a non object-oriented language close to Pascal. The QNAP2 code dealing with OCB is shorter than 1,000 lines.

The next version of OCB, which is currently in development, shall support multiple users viewed as processes in a very simple way to test the efficiency of concurrency control. As far as we know, Justitia is the only benchmark to have actually addressed this problem, though OO7 also has a multi-user version in development. OO1 was designed as multi-user, but the published results only involve a single user. One of our research objectives is to provide clear specifications for our benchmark so that others can readily implement it and provide feedback to improve it.

OCB Database

The OCB database is highly generic because it is rich, simple to achieve, and very tunable. It is made of a predefined number of classes (NC) derived from the same metaclass (Figure 5). A class has a unique logical identifier, Class_ID, and is defined by two parameters: $M A X N$ $R E F$, the maximum number of references in the class' instances; and BASESIZE, an increment size used to compute the InstanceSize after the inheritance graph is processed at database generation time. On Figure 5, note that the UML « bind » clause indicates that classes are instantiated from the metaclass using the parameters between brackets.

Since different references can point to the same class, 0-N, 1-N, and M-N links are implicitly modeled. Each of these CRef references has a type: TRef. There are NTREF different types of references. A reference type can be, for instance, a type of inheritance, aggregation, or user association. Eventually, an Iterator is maintained within each class to save references toward all its instances.

Objects in the database (instances of class $O B J E C T$ ) are characterized by a unique logical identifier $O I D$ and by their class through the ClassPtr pointer. Each object possesses $A T$ TRANGE integer attributes that may be read and updated by transactions. A string of size InstanceSize, the Filler, simulates the actual size the object should occupy on disk.

\footnotetext{
${ }^{2}$ http://eric.univ-lyon2.fr/ jdarmont, Software section
} 
After instantiating the database schema, an object $O$ of class $C$ points through the ORef references to at most MAXNREF objects. These objects are selected from the iterator of the class referenced by $C$ through the corresponding $C R e f$ reference. For each direct reference identified by an ORef link from an object $o_{i}$ toward an object $o_{j}$, there is also a backward reference (BackRef) from $o_{j}$ to $o_{i}$.

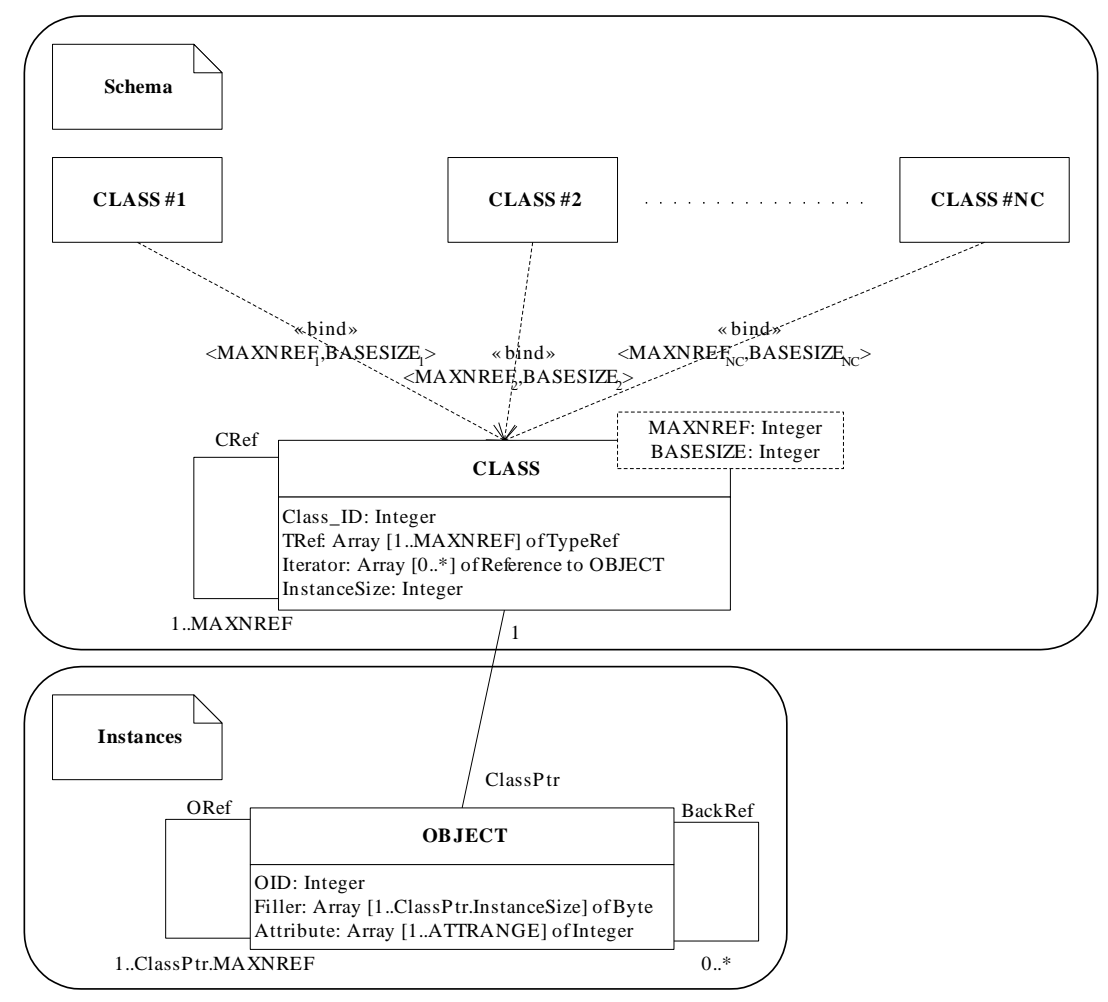

Figure 5: OCB database schema

The database generation proceeds through three primary steps.

1. Instantiation of the $C L A S S$ metaclass into $N C$ classes: creation of the classes without any reference, then selection of the classes referenced by each class. The type of the references (TRef) can either follow the DIST1 random distribution or be set up a priori. The referenced classes belong to the [Class_ID -CLOCREF,Class_ID + CLOCREF] interval that models a certain locality of reference, as introduced by OO1, but at the class level. The class reference selection can either follow the DIST2 random distribution or be set up a priori. NIL references are possible.

2. Database consistency check-up: suppression of all the cycles and discrepancies within the graphs that do not allow them, e.g., inheritance graphs or composition hierarchies. 
3. Instantiation of the $N C$ classes into $N O$ objects: creation of the objects, without any reference - their class follows the DIST3 random distribution, then random selection of the objects referenced by each object. The referenced objects belong to the $[O I D-O L O$ $C R E F, O I D+O L O C R E F]$ interval that models a certain locality of reference at the instance level. The random selection of object references follows the DIST4 random distribution. Reverse references (BackRef) are instantiated when the direct links are instantiated.

The random numbers are generated by the Lewis-Payne random generator (Lewis \& Payne, 1973), which is one of the best pseudorandom number generators currently available. The database parameters are summarized in Table 1.

\begin{tabular}{|l|l|c|}
\hline Parameter name & \multicolumn{1}{|c|}{ Parameter } & Default value \\
\hline NC & Number of classes in the database & 50 \\
\hline MAXNREF (i) & Maximum number of references, per class & 10 \\
\hline BASESIZE (i) & Instances base size, per class & 50 bytes \\
\hline NO & Total number of objects & 20,000 \\
\hline NREFT & Number of reference types (inheritance, aggregation, etc.) & 4 \\
\hline ATTRANGE & Number of integer attributes in an object & 1 \\
\hline CLOCREF & Class locality of reference & NC \\
\hline OLOCREF & Object locality of reference & NO \\
\hline MAXRETRY & Maximum number of retries when linking objects & 3 \\
\hline DIST1 & Reference types random distribution & Uniform \\
\hline DIST2 & Class references random distribution & Uniform \\
\hline DIST3 & Objects in classes random distribution & Uniform \\
\hline DIST4 & Objects references random distribution & Uniform \\
\hline
\end{tabular}

Table 1: OCB database parameters

\section{OCB Operations}

The core of the workload is organized around several transactions, the traversals, which are able to explore the effects of clustering. Several operations that do not benefit from any clustering effort have been re-introduced, e.g., creation and update operations. A full description of the benchmark's operations follows.

- Random Access: Access to NRND objects following the DIST5 random distribution.

- Sequential Scan: Browse the instances of a class following the DIST6 random distribution (Simple Scan). A Range Lookup additionally tests the value of NTEST attributes in each instance. 
- Traversals: Traversal operations are divided into two types: Set-Oriented Accesses (or Associative Accesses) and Navigational Accesses, which have been empirically found by Mc Iver (1994) to match breadth-first and depth-first traversals; respectively. Navigational Accesses are further divided into Simple, depth-first traversals, Hierarchy Traversals that always follow the same type of reference, and finally Stochastic Traversals that randomly select the next link to cross. Stochastic traversals effectively simulate the access patterns caused by real queries, according to Tsangaris \& Naughton (1992). An object bears at most MAXNREF references numbered from 1 to $M A X N R E F$. At each step of a stochastic traversal, the probability to follow reference number $N(N \in[1, M A X N R E F])$ is $\mathrm{p}(N)=$ $\frac{1}{2^{N}}$. Each type of traversal proceeds from a root object following the DIST7 random distribution and up to a predefined depth depending on the traversal type. All these transactions can be reversed to follow the links backward, "ascending" the graphs.

- Update: Update operations are also subdivided into different types. Schema Evolutions deal with individual insertion and deletion of Class objects. The class to be deleted follows the DIST8 random distribution. Database Evolutions deal with individual insertion and deletion of objects. The object to be deleted follows the DIST9 random distribution. Eventually, Attribute Updates allow attribute changes, either of random accessed objects (Random Update of NUPDT objects following the DISTA random distribution) or of instances of a class following the DISTB random distribution (Sequential Update).

The execution of transactions by each client (the benchmark is to be multi-user) is organized according to the following protocol:

1) cold run of $C O L D N$ transactions whose types are determined randomly according to predefined probabilities. The purpose of this step is to fill in the cache in order to observe the real, stationary behavior of the clustering algorithm implemented in the benchmarked system;

2) warm run of HOTN transactions.

A latency time THINK can be introduced between each transaction run. Furthermore, the whole benchmark execution may be replicated so that the same set of transactions is executed on different randomly-generated object bases. This feature allows the computation of mean values and confidence intervals, which are typically more significant than a single measurement. The OCB workload parameters are summarized in Table 2. 


\begin{tabular}{|l|l|c|}
\hline \multicolumn{1}{|c|}{ Parameter(s) name(s) } & \multicolumn{1}{c|}{ Parameter(s) } & Default value(s) \\
\hline NRND & Number of objects accessed in Random Accesses & 50 \\
\hline NTEST & Number of attributes tested in Range Lookups & 1 \\
\hline SETDEPTH, SIMDEPTH, & Depth: Set-oriented Access, Simple Traversal, & 3,3, \\
HIEDEPTH, STODEPTH & Hierarchy Traversal, Stochastic Traversal & 5,50 \\
\hline NUPDT & Number of updated objects in Random Updates & Uniform \\
\hline DIST5, & Random distribution law: Random Access objects, & \\
DIST6, DIST7, & Sequential Scan classes, Transaction root objects, & \\
DIST8, DIST9, & Schema Evolution classes, Database Evolution objects, & \\
DISTA, DISTB & Random Update objects, Sequential Update classes & $0.1,0.05$, \\
\hline PRND, PSCAN, & Occurrence probability: Random Access, Simple Scan, & $0.05,0.2,0.2$, \\
PRANGE, PSET, PSIMPLE, & Range Lookup, Set Access, Simple Traversal, & $0.2,0.1,0.005$, \\
PHIER, PSTOCH, & Hierarchy Traversal, Stochastic Traversal, & $0.005,0.02$, \\
PCINSERT, PCDEL, POINSERT,, & Class Insertion, Class Deletion, Object Insertion, & $0.02,0.025,0.025$ \\
PODEL, PRNDUP, PSEQUP & Object Deletion, Random Update, Sequential Update & 1,000 \\
\hline COLDN & Number of transactions executed during the cold run & 10,000 \\
\hline HOTN & Number of transactions executed during the warm run & 0 \\
\hline THINK & Average latency time between two transactions & 1 \\
\hline CLIENTN & Number of clients & Default seed \\
\hline RSEED & Random generator seed & 0.000 \\
\hline
\end{tabular}

Table 2 : OCB workload parameters

The metrics measured by OCB are basically:

- database response time (global and per transaction type) and throughput. In a client-server environment, times must be measured on the client side with standard system primitives like time () or getrusage () in $\mathrm{C}++$. The replication of the transactions compensates for the possible inaccuracy of these functions. If the number of transactions is sufficiently large, the absence of such system functions can be compensated by a manual timing, as it is specified for OO1;

- number of accessed objects (both globally and per transaction type). The computation of these usage statistics must be included in the benchmark's code;

- number of Input/Output (I/Os) performed. The I/Os necessary to execute the transactions and the I/Os needed to cluster the database (clustering overhead) must be distinguished. I/O usage can be obtained through the $\mathrm{C}++$ getrusage () function or by statistics provided by the DBMS. For instance, $\mathrm{O}_{2}$ provides such statistics.

Comparison of OCB to the Existing Benchmarks

\section{Genericity of $O C B$}


Since we intend to provide a generic benchmark, our tool must be able to model various types of databases and applications. In addition, it must also be able to imitate the demeanor of previous object-oriented benchmarks. Schreiber (1994) claims Justitia bestows this property provided the benchmark is properly parameterized. However, he does not provide any solid evidence to back up his claim.

We have shown that the OCB database is generic by comparing it to the object bases from existing benchmarks (Tables 3 and 4). In terms of workload, however, the demonstration of genericity is more difficult to achieve. OO7 especially offers a wide range of complex transactions. Some of them have been discarded when designing OCB, because they added complexity without providing much insight. Still, the transactional behavior of OO1, HyperModel, and Justitia can easily be imitated. Furthermore, some of OCB's operations, if combined, can be equivalent to OO7's complex operations.

\begin{tabular}{|l|c|c|}
\hline OCB parameter & OO1 & HyperModel \\
\hline NC & 2 & 3 \\
\hline MAXNREF (i) & Parts: 3 & 5 (Parent/Children) \\
& Connections: 2 (PartOf/Part) & + NO (RefTo/RefFrom) \\
& & +1 (Specialization) \\
\hline BASESIZE (i) & Parts: 50 bytes & Node: 20 bytes \\
& Connections: 50 bytes & TextNode: 1000 bytes \\
& & FormNode: 20008 bytes \\
\hline NO & 20000 parts & 3906 Nodes \\
& +60000 connections & +125 FormNodes \\
& & +15500 TextNodes \\
\hline NREFT & 3 & 4 \\
\hline CREFLOC & NC & NC \\
\hline OREFLOC & RefZne & Level $k+1$ in the Parent/Children hierarchy \\
\hline DIST1 & Constant (non random) & Constant (non random) \\
\hline DIST2 & Constant (non random) & Constant (non random) \\
\hline DIST3 & Constant (non random) & Constant (non random) \\
\hline DIST4 & Uniform & Uniform \\
\hline
\end{tabular}

Table 3: OCB tuning to imitate OOI and HyperModel object bases

\begin{tabular}{|l|c|c|}
\hline OCB parameter & OO7 & Justitia \\
\hline NC & 10 & 6 \\
\hline MAXNREF (i) & Design object: 0 & Database Entry: 0 \\
& Atomic part: 20 & Node: 2 \\
& Connection: 18 & CO: 3 \\
& Composite part: NumAtomicPerComp +8 & PO: PO_ATT_SIZE +3 \\
& Document: 1 & \\
& Manual: 1 & \\
& Assembly: 2 & \\
& Complex assembly: NumAssmPerAssm +2 & \\
& Base assembly: NumComPerAssm $\mathrm{x} 2+1$ & \\
\hline
\end{tabular}




\begin{tabular}{|c|c|c|}
\hline & Module: $\quad \sum_{i=0}^{\text {NumAssmLevels }}$ NumAssmPeAssm ${ }^{i}$ & \\
\hline BASESIZE (i) & $\begin{array}{c}\text { Design object: } 18 \text { bytes } \\
\text { Atomic part: } 12 \text { bytes } \\
\text { Connection: } 14 \text { bytes } \\
\text { Composite part: } 0 \\
\text { Document: DocumentSize }+44 \text { bytes } \\
\text { Manual: ManualSize }+48 \text { bytes } \\
\text { Assembly: } 0 \\
\text { Complex assembly: } 0 \\
\text { Base assembly: } 0 \\
\text { Module }: 0\end{array}$ & $\begin{array}{c}\text { Database entry: } 4 \text { bytes } \\
\text { PO: } 0 \\
\text { Node: } 4 \text { bytes } \\
\text { CO: } 0 \\
\text { DO: DO_ATT_SIZE bytes } \\
\text { SO:SO_ATT_SIZE bytes }\end{array}$ \\
\hline NO & \begin{tabular}{c}
\multicolumn{2}{c}{$\begin{array}{c}\text { NumModules modules } \\
+ \text { NumModules manuals }\end{array}$} \\
$+\sum_{i=0}^{\text {NumAssmLev els-1 }}$ NumAssmPeAssm complex assemblies \\
+ NumPerAssm ${ }^{\text {NumAssmLevels }}$ base assemblies \\
+ NumCompPerModule composite parts \\
+ NumCompPerModule documents \\
+ NumAtomicPerComp . NumCompPerModule atomic \\
parts \\
+ NumAtomicPerComp . NumCompPerModule . Num- \\
ConnPerAtomic connections \\
\end{tabular} & $\begin{array}{c}\text { SECTION . MAXWIDTH } \\
\text { MAXLEVEL }\end{array}$ \\
\hline NREFT & 12 & 3 \\
\hline CREFLOC & $N C$ & $N C$ \\
\hline OREFLOC & $N O$ & $N O$ \\
\hline DIST1 & Constant (non random) & Constant (non random) \\
\hline DIST2 & Constant (non random) & Constant (non random) \\
\hline DIST3 & Constant (non random) & Constant (non random) \\
\hline DIST4 & Constant + Uniform & Constant (non random) \\
\hline
\end{tabular}

Table 4: OCB tuning to imitate $0 O 7$ and Justitia object bases

\section{Comparison with Gray's Criteria}

Gray (1993) defines four primary criteria concerning the specification of a good benchmark:

1) relevance: it must concern aspects of performance that appeal to the largest number of potential users;

2) portability: it must be reusable to test the performances of different OODBs;

3) simplicity: it must be feasible and must not require too many resources;

4) scalability: it must be able to be adapted to small or large computer systems, or new architectures.

When designing OCB, we mainly intended to palliate two shortcomings in existing benchmarks: their lack of genericity and their inability to properly evaluate the performances of object clustering techniques. To achieve this goal, we designed a fully tunable benchmark, al- 
lowing it either to be generic or to be specialized for a given purpose. The consequences of this choice on Gray's criteria are the following:

- relevance: as previously stated, all the transactions from existing benchmarks have been included in OCB except the most intricate operations from OO7;

- portability: $\mathrm{OCB}$ has been used to evaluate the performances of the $\mathrm{O}_{2}$ and the Texas systems. Both these implementations have been made in $\mathrm{C}++$. OCB has also been included in simulation models written in QNAP2 and a simulation package called DESP-C++. OCB's code is short and simple in all these cases;

- simplicity: complete specifications for our benchmark are provided in this section in order to support understandability and ease of implementation;

- scalability: OCB is a very flexible benchmark due to an extensive set of parameters. Its object base can take different sizes and complexity levels and its various transactions can model a fair number of applications.

The characteristics of the existing benchmarks and OCB according to these criteria are summarized in Table 5.

\begin{tabular}{|l|c|c|c|c|}
\cline { 2 - 5 } \multicolumn{1}{c|}{} & Relevance & Portability & Simplicity & Scalability \\
\hline OO1 & -- & ++ & ++ & - \\
\hline HyperModel & + & + & - & -- \\
\hline OO7 & ++ & + & - & - \\
\hline Justitia & - & -- & + & + \\
\hline OCB & ++ & + & + & ++ \\
\hline \multicolumn{2}{r|}{ Strong point : + } \\
\end{tabular}

Table 5: Comparison of existing benchmarks to $O C B$

\section{VALIDATION EXPERIMENTS}

We present in this section performance evaluations performed with OCB on the $\mathrm{O}_{2}$ OODB, the Texas persistent object store, and the DSTC and DRO clustering techniques, which are implemented in Texas. Our research objective did not include a comparison of the performances of $\mathrm{O}_{2}$ and Texas. This would have been troublesome since our versions of these systems did not run on the same platform. Furthermore, $\mathrm{O}_{2}$ and Texas are quite different in their philosophy and functionalities. $\mathrm{O}_{2}$ is a full OODB supporting concurrent and secure accesses while Texas is positioned as an efficient persistent store for $\mathrm{C}++$. We only intended to show that OCB provided valid performance evaluations. 
Since we recommended the use of a complex object base, the feasibility of our specifications has been checked by measuring the database average generation time function of the database size (number of classes and number of instances). For schemas containing 10, 20, and 50 classes, the number of instances $N O$ was varied from 5,000 to 50,000. The actual database size was also measured for all these configurations.

Next, the object base configuration was varied: number of classes $N C$, number of instances $N O$, number of inter-object references $M A X N R E F$. Four database configurations were obtained using $N C$ values of 20 and 50, and MAXNREF values of 5 and 10 . Then, the number of instances in the database was varied from 500 to 20,000 for each configuration. The scope of this study is limited to raw performance results, i.e., the average response time and the average number of I/Os necessary to execute the operations.

The efficiency of the DSTC and DRO clustering techniques has been compared by measuring the performances achieved by Texas before and after object clustering. To achieve the comparison, we used a mid-sized OCB database composed of 50 classes and 100,000 objects, for a size of about $62 \mathrm{MB}$. The other OCB parameters defining the database were set to default. A series of 10,000 standard OCB transactions was executed on this database, before and after object clustering. System performance was measured in terms of I/Os, response time, and relative performance improvement due to clustering. Only the results concerning I/Os are presented here because response time plots present exactly the same tendencies and do not bring additional insight. Eventually, these experiments have been performed in several memory configurations. Since Texas makes an intensive use of virtual memory, it was interesting to see how the system behaved when the ratio main memory size / database size varied. The whole process was reiterated 100 times so that mean tendencies could be achieved. In each iteration, the same random seed was selected for the DSTC and DRO experiments so that they were rigorously identical.

Results for $\mathrm{O}_{2}$

\section{Material Conditions}

The $\mathrm{O}_{2}$ server (version 5.0) was installed on an IBM RISC $600043 \mathrm{P} 240$ biprocessor workstation. Each processor was a Power PC 604e 166. The workstation had 1 GB ECC RAM. The operating system was AIX version 4 . The $\mathrm{O}_{2}$ server cache size was $16 \mathrm{MB}$ by default. 


\section{Object Base Generation}

Figure 6 displays the database generation time function of the number of classes and the number of instances in the base. It shows that generation time increased linearly when the schema was made of 10 or 20 classes. The increase was more accentuated with 50 classes, because when the $\mathrm{O}_{2}$ client cache was full, which happened with the biggest databases, an execution error occurred. To fix this problem, the generation process has been marked out with commits. These multiple commits were more costly than a single validation at the end of the generation process. The feasibility of OCB was also demonstrated, since in the worst case generation time was less than one hour. Moreover, a given object base could be saved and reused multiple times so that the generation process could be avoided each time.

Figure 7 shows how the size of the randomly generated database linearly evolved with the number of classes and instances. Hence, it was easy to foresee the final size of a database when setting the $N C$ and $N O$ parameters. The default OCB database (50 classes, 20,000 instances) had a mean size of $30 \mathrm{MB}$, which is average for a benchmark. For instance, the large database in $\mathrm{OO} 1$ has a size of $40 \mathrm{MB}$. However, we showed that larger databases are possible.

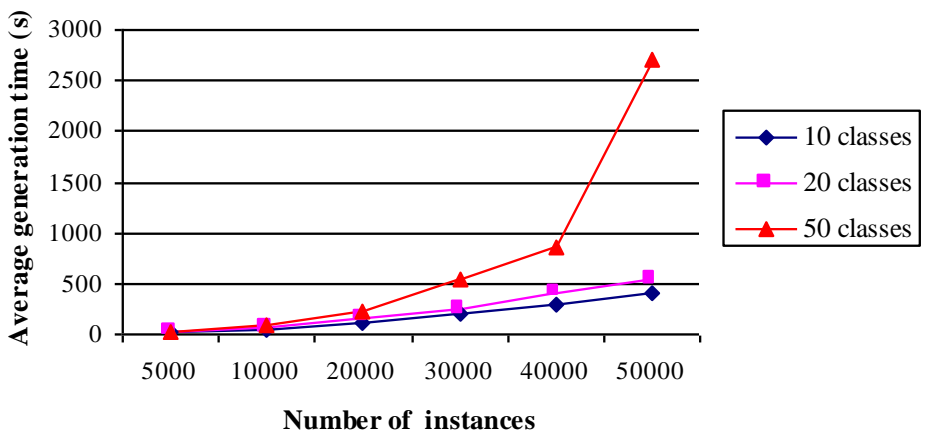

Figure 6: Database generation time $\left(\mathrm{O}_{2}\right)$ 


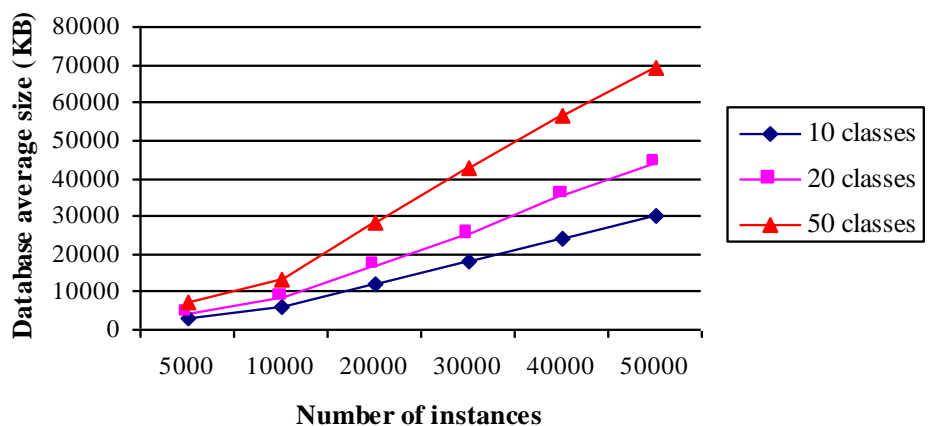

Figure 7: Actual database size $\left(\mathrm{O}_{2}\right)$

\section{Object Base Usage}

In Figure 8, we plotted the mean number of I/Os globally necessary to execute the transactions function of the number of instances in the object base $(N O)$ for our four database configurations. We did the same in Figure 9 for the mean response time.

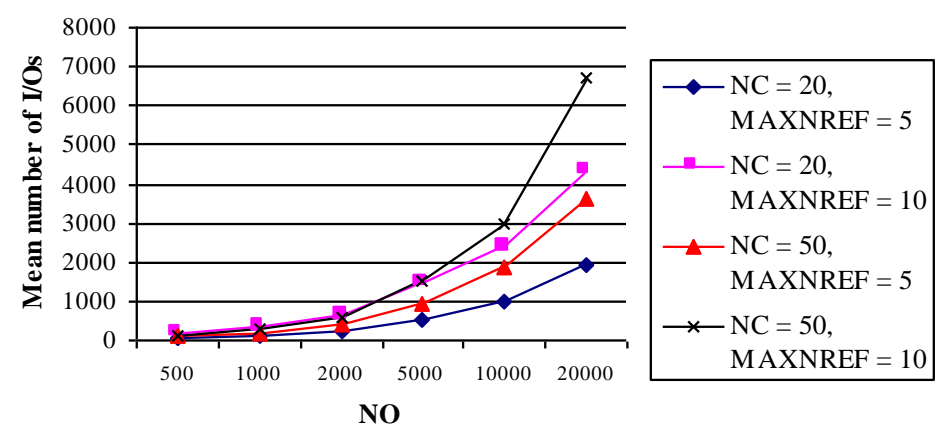

Figure 8: Mean number of I/Os $\left(\mathrm{O}_{2}\right)$

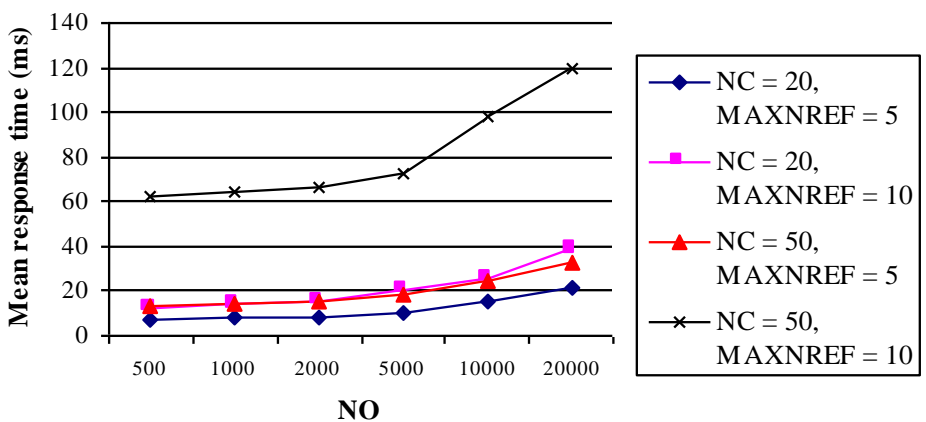

Figure 9: Mean response time $\left(\mathrm{O}_{2}\right)$

We can see that the performances of $\mathrm{O}_{2}$ logically decreased in the three following cases. 
- $N C$ increase - This was due to the structure of the OCB schema. The more classes it contained, the deeper the inheritance graph was. Since information is inherited at each level from the upper level, leaf classes in the inheritance graph have bigger instances than root classes. Hence, a higher number of classes induced bigger object sizes, so the database occupied more disk pages.

- MAXNREF increase - The number of objects accessed by transactions that browsed all the references increased.

- NO increase - The database got bigger and objects were distributed over more disk pages.

The evolution of our two performance criteria was quite similar. This result was expected, since most treatments performed by the system when running OCB deal with loading objects from disk.

Results for Texas

\section{Material Conditions}

Texas version 0.5 was installed on a PC Pentium-II 266 with a 64 MB SDRAM. The host operating system was Linux 2.0.30. The swap partition size was $64 \mathrm{MB}$. Texas has been compiled with the GNU C++ compiler version 2.7.2.1.

\section{Object Base Generation}

Figure 10 displays the average time for database generation function of the number of instances and the number of classes in the database. It shows that generation time did not increase linearly. However, the longest generation times were approximately 10 minutes long, which was an acceptable rate.

Texas did not appear to have the same behavior than $\mathrm{O}_{2}$ because average generation time was greater when the schema contained few classes. This result can be attributed to two phenomena.

- The graph consistency check for acyclic graphs was more complex when the number of classes was low. In these conditions, the interclass references were dispersed in a reduced class interval and formed very dense graphs. 
- When the database did not fit wholly into the main memory, the system swapped, which was costly both in terms of I/Os and time.

The actual size of the object bases generated with Texas was always less than $60 \mathrm{MB}$, as shown in Figure 12, allowing them to be stored in the 64 MB memory. Hence, the graph consistency check was prevalent while in the case of $\mathrm{O}_{2}$, swap was prevalent. This hypothesis has been checked with Texas by reducing the available memory under Linux to $16 \mathrm{MB}$. Figure 11 displays the results of these tests, which confirmed our assumption.

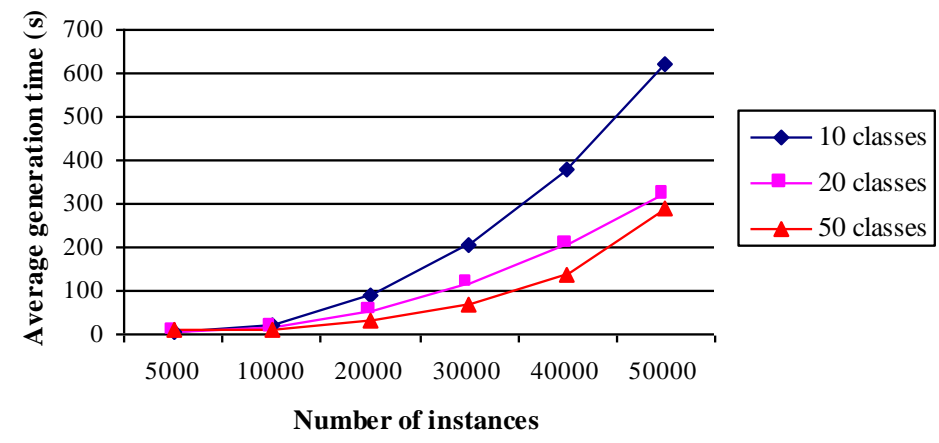

Figure 10: Database generation time (Texas)

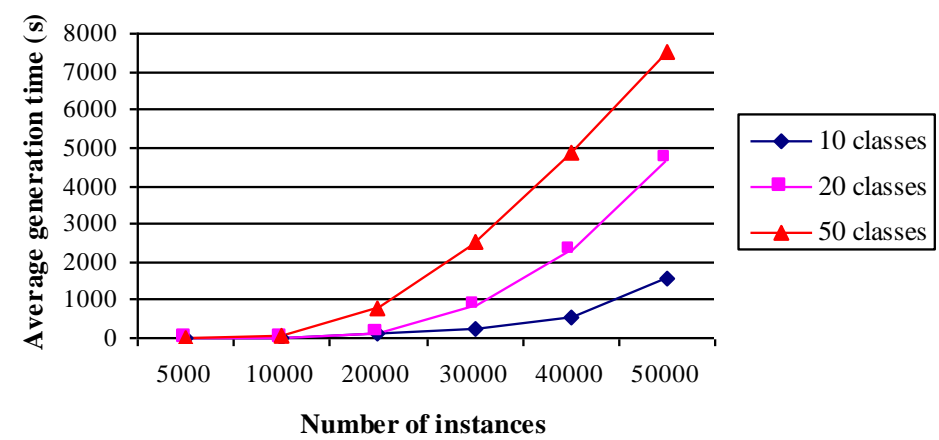

Figure 11: DB generation time with $16 \mathrm{MB}$ memory

Figure 12 eventually shows how the database real size evolved with the number of instances and the number of classes in the database. As happened with $\mathrm{O}_{2}$, this evolution was linear. The average database size was about $20 \mathrm{MB}$ with Texas. The object bases generated with $\mathrm{O}_{2}$ were one third bigger due to the objects storage format: Texas directly uses the memory format while $\mathrm{O}_{2}$ uses the WiSS (Chou, 1985) record structures that are more elaborate. 


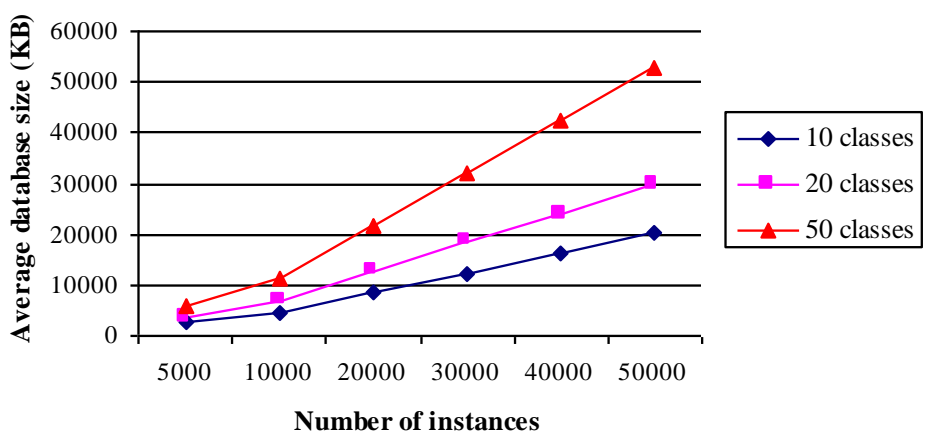

Figure 12: Actual database size (Texas)

\section{Object Base Usage}

In Figure 13, we plotted the mean number of I/Os globally necessary to execute the transactions function of the number of instances in the object base $(N O)$, for our four database configurations. We did the same in Figure 14 for the mean response time.

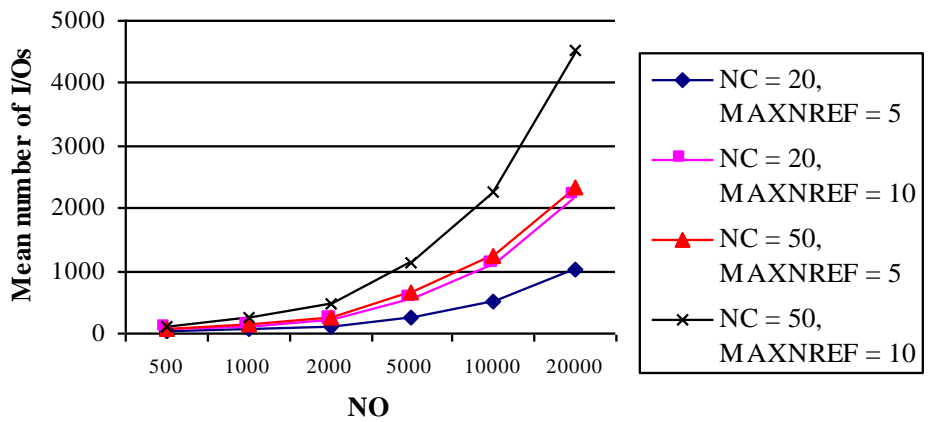

Figure 13: Mean number of I/Os (Texas)

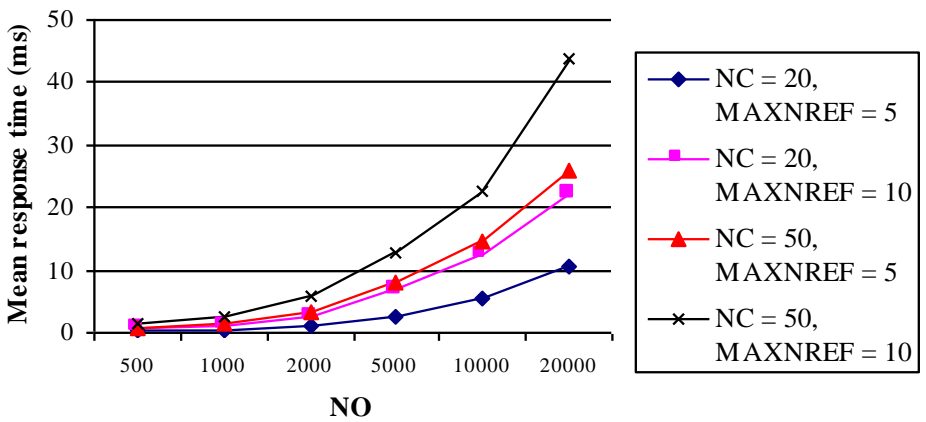

Figure 14: Mean response time (Texas) 
In the case of Texas, the correlation between the mean number of I/Os and the mean response time appeared tighter than for $\mathrm{O}_{2} \cdot \mathrm{O}_{2}$ indeed includes many more features than Texas (security, concurrency control, and others) that add an overhead that is not directly linked to disk accesses.

Clustering Effect Evaluation

\section{Results for DSTC}

Figures 15 and 16 show that clustering with DSTC indeed allows a significant gain in performance, especially when the amount of main memory available is small. Clustering is definitely more useful when the database does not fit wholly within the main memory, since its effects are felt as soon as the system swaps and not only at page load time. This assumption is neatly confirmed by the clustering gain factor graph in Figure 16. Clustering gain factor is equal to the number of I/Os necessary to execute the transactions after clustering divided by the number of I/Os necessary to execute the transactions before clustering.

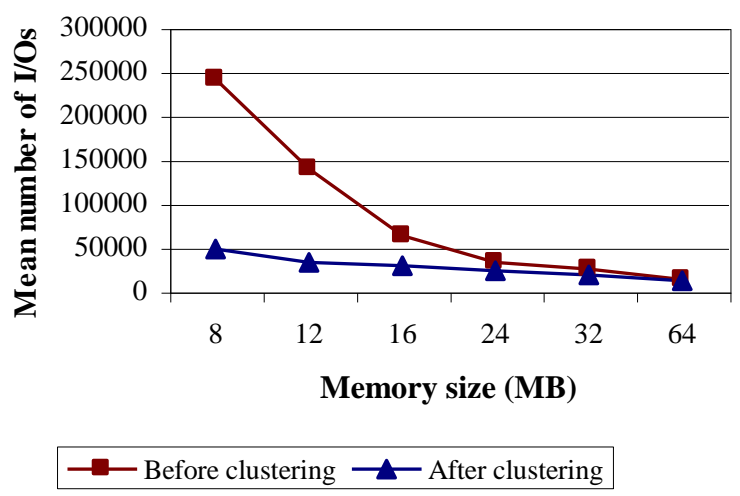

Figure 15: Mean number of I/Os (DSTC) 


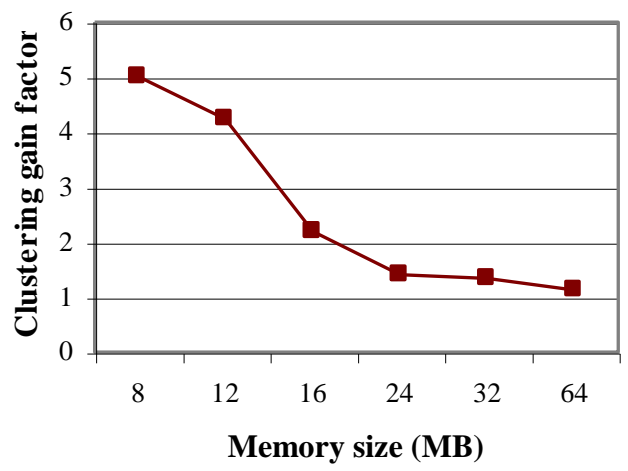

Figure 16: Clustering gain factor (DSTC)

\section{Results for DRO}

Figures 17 and 18 show that DRO bears the same overall behavior as DSTC. However, the gain factor achieved with DRO looks much better. It is indeed about 15 . The comparison is unfair, though, because we selected the optimal set of parameters for DRO clustering, while we could not do it for DSTC. Due to technical problems with big databases, we had to parameterize DSTC so that clustering was not the best possible. There was a threshold effect on a set of DSTC parameters. Below this "threshold", everything worked out fine but clustering was average. Beyond the "threshold", clustering units were too big for Texas to manage and the system invariably crashed.

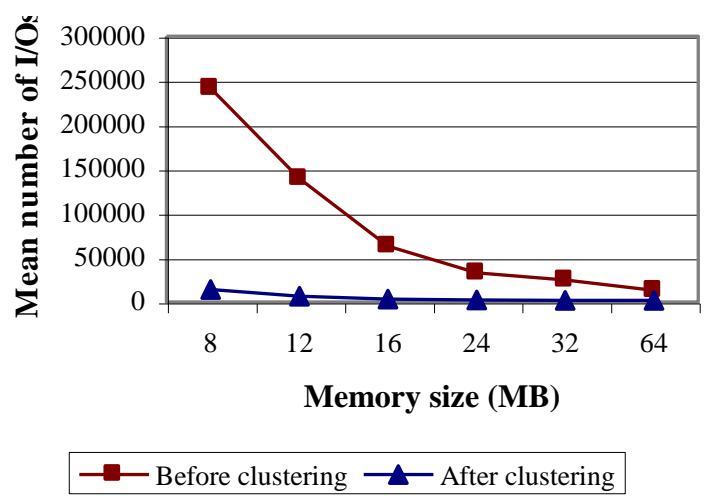

Figure 17: Mean number of I/Os (DRO) 


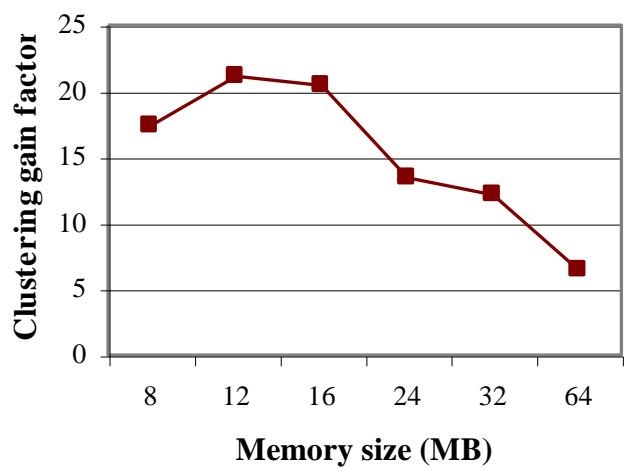

Figure 18: Clustering gain factor (DRO)

\section{Comparison of DSTC and DRO}

To eventually compare DSTC and DRO on a fair ground, we used a smaller database so that DSTC could properly work. We used OCB's default database (50 classes, 20,000 instances, about $20 \mathrm{MB}$ ) and ran two series of typical transactions that were likely to benefit from clustering: depth-3 hierarchy traversals (that always follow the same type of reference) and depth2 simple traversals (depth-first traversals). The depth of traversals was reduced regarding OCB's default parameters so that the generated clusters were not too big and the effects of clustering were clear. The traversals have been performed from 100 predefined root objects and each of them was executed 10 times.

Table 6 displays the mean number of I/Os concerning database usage before and after clustering. It shows that DSTC and DRO both achieve a substantial increase in performance (factor 6-7). DRO looks even better, though more tuning with DSTC should bring this method on the same level. Unfortunately, such tuning still provoked execution errors in Texas. The big difference between DSTC and DRO lies in clustering overhead (the number of I/Os necessary for an algorithm to cluster the database). DSTC induces a high overhead, which renders it difficult to implement truly dynamically. Its authors actually advocate its triggering when the database is idle. On the contrary, DRO, which is much simpler, presents a lower overhead (about 4 times lower) and is certainly better suited to a dynamic execution.

\begin{tabular}{|c|c|c|c|c|}
\cline { 2 - 5 } \multicolumn{1}{c|}{} & \multicolumn{2}{c|}{ Hierarchy traversals } & \multicolumn{2}{c|}{ Simple traversals } \\
\cline { 2 - 5 } \multicolumn{1}{c|}{} & $\boldsymbol{D S T C}$ & $\boldsymbol{D R O}$ & $\boldsymbol{D S T C}$ & $\boldsymbol{D R O}$ \\
\hline Pre-clustering usage & 1682.6 & 1686 & 1682 & 1683 \\
\hline Post-clustering usage & 270.8 & 226 & 281.75 & 236.75 \\
\hline Clustering gain factor & 6.21 & 7.46 & 5.97 & 7.11 \\
\hline Clustering overhead & 12219.4 & 3286.8 & 12174 & 2804.5 \\
\hline
\end{tabular}




\section{FUTURE TRENDS}

Future research about OODB benchmarking could take at least three directions. First, directly using a benchmark such as OCB with its default parameters for performance analysis of clustering and pre-fetching algorithms may produce misleading results, since such optimization methods are particularly sensitive to the object base traversal order, both within and between transactions. Indeed, there often exists dependencies between successive transactions. To handle this issue, a "dynamic" extension to the OCB benchmark called DOCB has recently been designed (He \& Darmont, 2001). It features various inter-traversal dependencies and changes in access pattern.

Second, we only exposed the principles of a multi-user version of the OCB benchmark. The transition from the single-user version toward an operational multi-user version is not immediate and requires a particular care. The aim of this evolution is to evaluate the efficiency of concurrency control and to see how systems react when faced to a more important and heterogeneous workload. Since OODBs normally operate in a concurrent environment, their performances cannot be gauged with a single-user benchmark.

Third, one very different aspect we did not consider here is the "qualitative" element that is important to take into account when selecting an OODB. Atkinson, Birnie, Jackson, and Philbrow (1992), Banerjee and Gardner (1995), Kempe, Kowarschick, Kießling, Hitzelgerger, and Dutkowski (1995) all insist on the fact that functionality is at least as important as raw performances. Hence, criteria concerning these functionalities should be worked out. Sheer performance could be viewed as one of these criteria. Concerning optimization methods, we could, for instance, evaluate if a clustering heuristic's parameters are easy to apprehend and set up or if the algorithm is easy to use or transparent to the user.

Eventually, another point that can be considered is the adequacy of OCB to evaluate the performances of object-relational systems. OCB's generic model can of course be implemented with an object-relational system and most the operations are relevant for such a system. Thus, OCB can allow the comparison of different logical or physical organizations (distribution of the objects into tables, implementation of associations by values or by pointers, distribution of tables into tablespaces, index...). OCB can be considered as a candidate benchmark for this 
type of systems, even if extensions are needed to take into account additional aspects, regarding Abstract Data Types, in particular.

\section{CONCLUSION}

The full specifications for the OCB object-oriented benchmark have been presented. OCB's main qualities are its richness, its flexibility, and its compactness. This benchmark indeed offers an object base whose complexity has never been achieved before in object-oriented benchmarks. Furthermore, since this database and likewise the transactions running on it are wholly tunable through a collection of comprehensive but easily set parameters, OCB can be used to model many kinds of object-oriented database applications. Eventually, OCB's code is short, reasonably easy to implement, and easily portable.

We have shown this benchmark was merely feasible by measuring generation time for its random database. It appears that in the worst case, an OCB object base is generated in less than one hour, which is quite acceptable. Furthermore, the largest databases can be saved for multiple uses.

We have also illustrated the genericity of the benchmark by showing how it could imitate both the schema and the operations of four existing benchmarks. The flaws identified in these previous benchmarks have been underlined and an attempt was made to correct them. We eventually demonstrated that OCB could be used as a general-purpose benchmark by evaluating the performances of the $\mathrm{O}_{2}$ OODB and the Texas persistent object store. We also showed it could serve as a more specialized benchmark by comparing the effects of the DSTC and DRO clustering methods on the performances of Texas.

\section{REFERENCES}

Anderson, T.L., Berre, A.J., Mallison, M., Porter, H.H., \& Scheider, B. (1990). The HyperModel Benchmark. International Conference on Extending Database Technology, Venice, Italy. 317-331.

Atkinson, M.P., Birnie, A., Jackson, N., \& Philbrow, P.C. (1992). Measuring Persistent Object Systems. $5^{\text {th }}$ International Workshop on Persistent Object Systems, San Miniato (Pisa), Italy. 63-85.

Banerjee, S., \& Gardner, C. (1995). Towards An Improved Evaluation Metric For Object Database Management Systems. OOPSLA '95 Workshop on Object Database Behavior, Benchmarks and Performance, Austin, Texas. 
Benzaken, V. (1990). An Evaluation Model for Clustering Strategies in the $\mathrm{O}_{2}$ ObjectOriented Database System. $3^{\text {rd }}$ International Conference on Database Theory, Paris, France. 126-140.

Bullat, F., \& Schneider, M. (1996). Dynamic Clustering in Object Database Exploiting Effective Use of Relationships Between Objects. ECOOP '96, Linz, Austria. Lecture Notes in Computer Science. 1098, 344-365.

Carey, M.J., Dewitt, D.J., \& Naughton, J.F. (1993). The OO7 Benchmark. ACM SIGMOD International Conference on Management of Data, Washington DC. 12-21.

Carey, M.J., Dewitt, D.J., Kant, C., \& Naughton, J.F. (1994). A Status Report on the OO7 OODBMS Benchmarking Effort. SIGPLAN Notices. 29(10), 414-426.

Cattell, R.G.G. (1991). An Engineering Database Benchmark. The Benchmark Handbook for Database Transaction Processing Systems, Jim Gray, Ed. Morgan Kaufmann. 247-281.

Chou, H.-T. (1985). Design and implementation of the Wisconsin storage system. Software Practice and Experience. 15(10).

Darmont, J., Petit, B., \& Schneider, M. (1998). OCB: A Generic Benchmark to Evaluate the Performances of Object-Oriented Database Systems. $6^{\text {th }}$ International Conference on Extending Database Technology (EDBT '98), Valencia, Spain. Lecture Notes in Computer Science. 1377, 326-340.

Darmont, J., Fromantin, C., Régnier, S., Gruenwald, L., \& Schneider, M. (2000). Dynamic Clustering in Object-Oriented Databases: An Advocacy for Simplicity. ECOOP 2000 Symposium on Objects and Databases, Sophia Antipolis, France. Lecture Notes in Computer Science. 1944, 71-85.

Deux, O. (1991). The $\mathrm{O}_{2}$ System. Communications of the ACM. 34(10), 34-48.

Gardarin, G., Gruser, J.-R., \& Tang, Z.-H. (1995). A Cost Model for Clustered ObjectOriented Databases, $21^{\text {st }}$ International Conference on Very Large Data Bases (VLDB '95), Zurich, Switzerland. 323-334.

Gray, J., Ed. (1993). The Benchmark Handbook for Database and Transaction Processing Systems $2^{\text {nd }}$ edition. Morgan Kaufmann.

He, Z., \& Darmont, J. (2001). Dynamic Object Clustering Benchmark (DOCB). Technical report. The Autralian National University, Australia; The University of Lyon 2, France.

Kempe, J., Kowarschick, W., Kießling, W., Hitzelgerger, R., \& Dutkowski, F. (1995). Benchmarking Object-Oriented Database Systems for CAD. $6^{\text {th }}$ International Conference on Database and Expert Systems Applications (DEXA '95), London, UK. Lecture Notes in Computer Science. 978, 167-176.

Lewis, T.G., \& Payne, W.H. (1973). Generalized feedback shift register pseudorandom number algorithm. Journal ACM. 20(3), 456-468.

Mc Iver, W.J., \& King, R. (1994). Self-Adaptive, On-Line Reclustering of Complex Object Data. ACM SIGMOD Conference, Minneapolis, Minnesota. 407-418.

Schreiber, H. (1994). JUSTITIA: a generic benchmark for the OODBMS selection. $4^{\text {th }}$ International Conference on Data and Knowledge Systems in Manufacturing and Engineering, Shatin, Hong Kong. 324-331. 
Singhal, V., Kakkad, S.V., \& Wilson, P.R. (1992). Texas: An Efficient, Portable Persistent Store. $5^{\text {th }}$ International Workshop on Persistent Object Systems, San Miniato, Italy.

Tiwary, A., Narasayya, V.R., \& Levy, H.M. (1995). Evaluation of OO7 as a system and an application benchmark. OOPSLA '95 Workshop on Object Database Behavior, Benchmarks and Performance, Austin, Texas.

Tsangaris, M.M., \& Naughton, J.F. (1992). On the Performance of Object Clustering Techniques. ACM SIGMOD International Conference on Management of Data, San Diego, California. 144-153. 